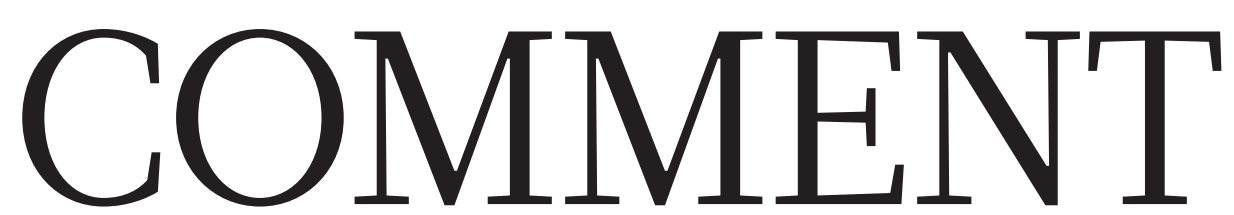

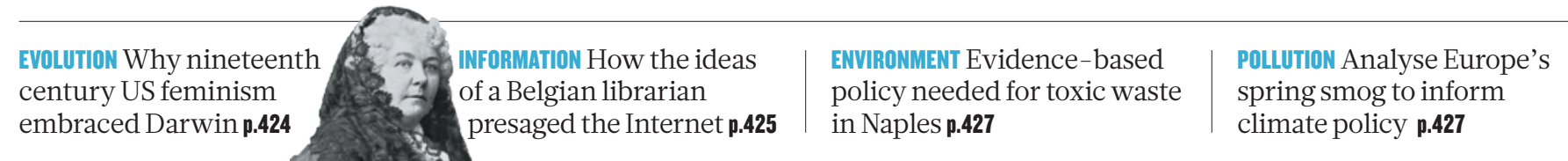

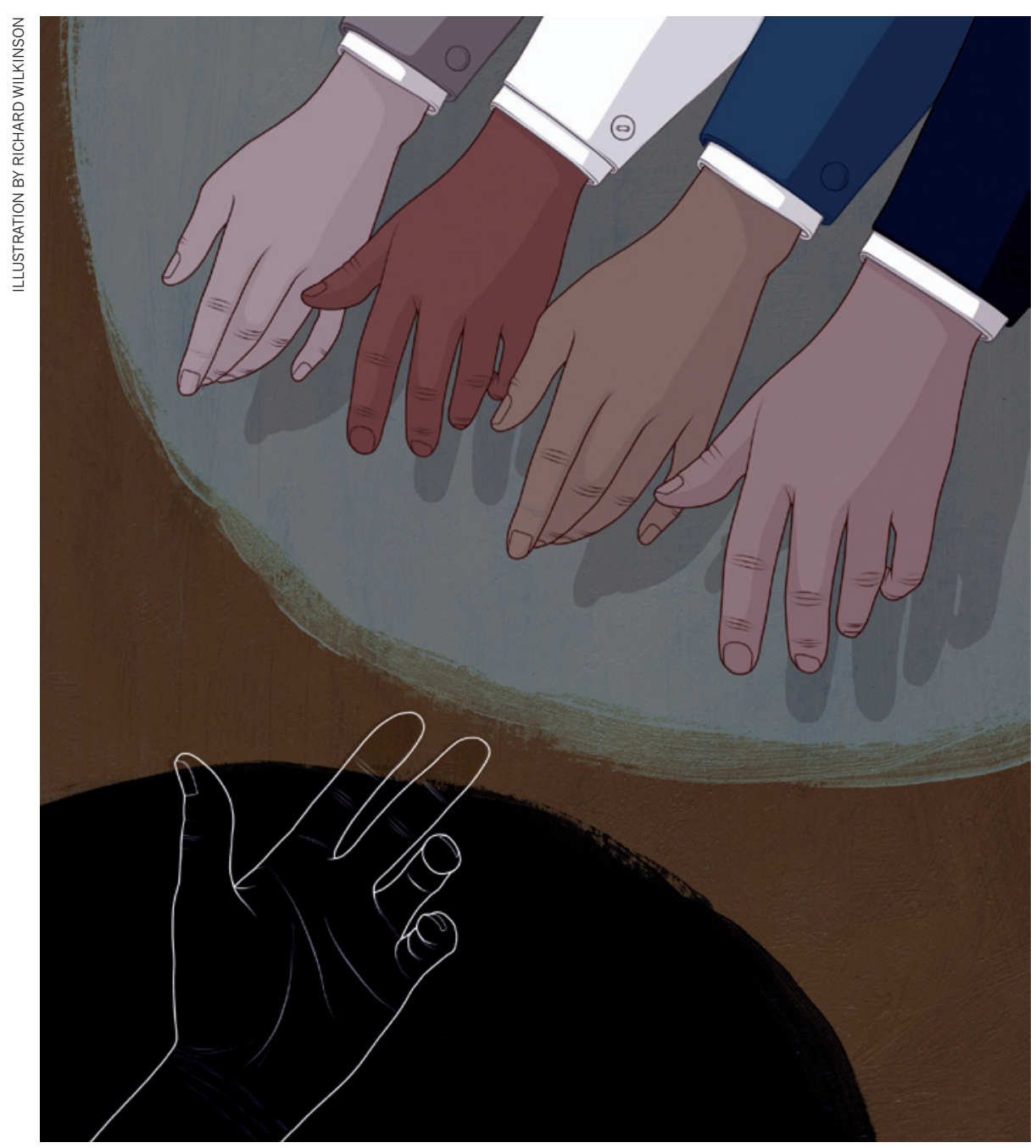

A ccording to the World Health Organization, almost 1 million people kill themselves every year. That is more than the number that die in homicides and war combined. A further 10 million to 20 million people attempt it.

Suicide is one of the three leading causes of death in the economically most productive age group - those aged 15-44 years - and rates have risen since the economic crisis triggered by the banking crash in 2008 (see 'Suicide rates in Europe'). For example, the number of suicides per year in the Netherlands rose by $30 \%$ between 2008 and 2012, from 1,353 to 1,753 . In the United States, the average suicide costs society US\$1.06 million according to the US Centers for Disease Control and Prevention.

Despite its enormous societal impact, little progress has been made in the scientific understanding or treatment of suicidal behaviour. We do know that up to $90 \%$ of suicides occur in people with a clinically diagnosable psychiatric disorder ${ }^{1}$. Large epidemiological studies have shown mental disorders, particularly depression and alcohol addiction, to be major risk factors ${ }^{2}$. And there is compelling evidence that adequate prevention and treatment of such disorders can reduce suicide rates ${ }^{3}$.

But psychiatry has long neglected the topic. Other than as symptoms of borderline personality disorder and mood disorders, suicide, suicide attempts and suicidal thoughts were not listed in the fourth edition of the Diagnostic and Statistical Manual of Mental Disorders (DSM-IV). The DSM-5 (published last year) does not code suicidal behaviour - the most prominent emergency in psychiatry in primary care. Suicidality is perceived as a medical complication rather than as a disorder in its own right.

For every study on suicidality published in the two highest-ranking general psychiatry journals (American Journal of Psychiatry and JAMA Psychiatry) over the past five years, there were six papers on schizophrenia, the incidence of which is one-quarter that of suicidal behaviour. And, in contrast to the studies on schizophrenia, those on suicidal behaviour are mostly epidemiological and do not investigate underlying mechanisms.

The lack of suicide research may be due to several factors. The first is cultural taboos. People are hesitant to talk about the suicide $>$ 
of a family member or friend, and many religions consider suicide dishonourable. It is often deemed unlawful, too. In India and Singapore, for example, attempted suicide is punishable by up to a year in prison. In several US states, suicide is still considered an unwritten 'common law' crime, which can have financial consequences for the family. Assisted suicide is illegal in many countries.

Second, the triggers of suicide are complicated, involving mental-health, financial, social, cultural and moral issues. Third, suicidal behaviour could be difficult to study if non-fatal suicidal attempts differ in aetiology from fatal ones.

What is urgently needed is a road map for the systematic study of the mechanisms of suicidal behaviour, independent of any associated disorder. Only then can evidencebased prevention programmes be framed.

In our view, these four steps are needed.

\section{FOUR-POINT PLAN}

Define suicide as a distinct disorder. Treatment of the mental disorders commonly associated with suicide, such as depression, fails to prevent suicidal behaviour in most people. Although suicidality touches different medical and psychosocial disciplines, it should come under the remit of psychiatry, which being at the crossroads of mind and brain is well positioned to appraise all dimensions. Psychiatry should take responsibility for defining suicidality adequately, incorporating it in classification systems, developing ratings scales to predict and assess severity and examining treatment options. This will make suicidal behaviour visible as a mental disorder.

Understand the mechanisms. The roots of suicide - psychological and neurobiological

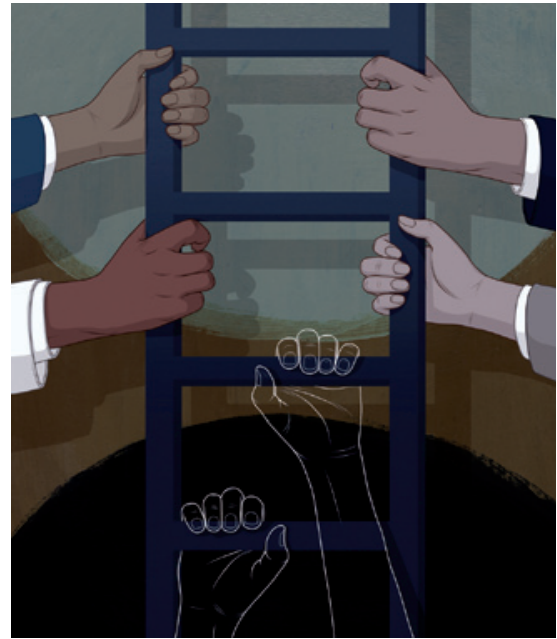

- could stem from difficulties in emotion regulation and the underlying brain circuits. The most important psychological correlates are anxiety, reduced impulse control and increased aggression ${ }^{2}$. Furthermore, people showing suicidal behaviour tend to suppress their emotions and have difficulties identifying their feelings ${ }^{4}$. Suicidality is associated with hopelessness, sensitivity towards social disapproval and a reduced ability to imagine positive future events ${ }^{5}$. But there are probably several routes to suicidal behaviour.

Research should focus on individual differences in cognitive control of emotion. Some people may have excessively strong emotional reactions to challenging events such as bereavement or job loss, some may lack cognitive flexibility and coping skills in the face of adversity, and some may show impulsive aggressive tendencies. Widely accepted models of the role of emotional reactivity and lack of cognitive control in setting the stage for suicidal behaviour need rigorous investigation ${ }^{6}$.

\section{SUICIDE RATES IN EUROPE}

In many nations, deaths from suicide increased after the economic crisis

triggered by the banking crash in 2008

\section{0 data}

Deaths from suicide per 100,000 inhabitants adjusted to a standard age distribution.

3.3-6.8

6.9-11.7

-11.8-13.7

-13.8-17.8

-17.9-32.9

No data

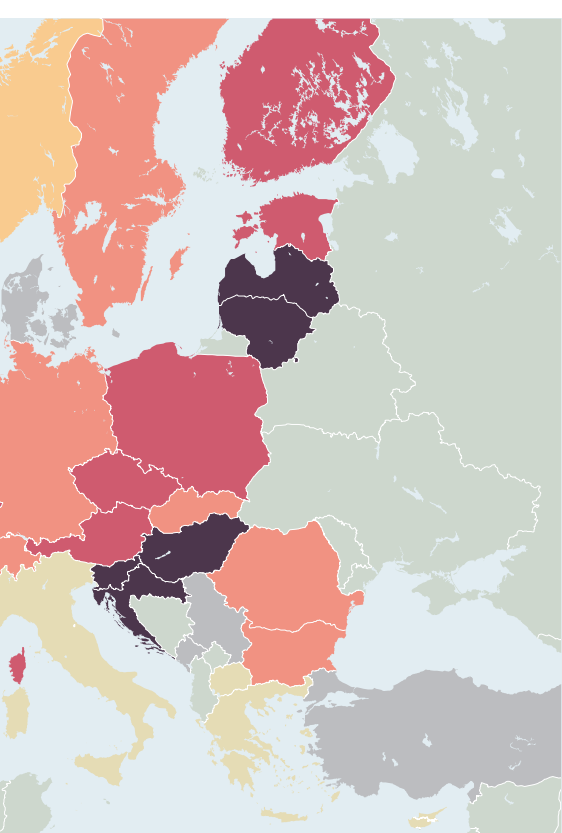

An example of the sort of research that we need more of is a neuroimaging study ${ }^{7}$ by a team led by psychiatrist Scott Matthews at the VA San Diego Healthcare System in California. They compared brain activity in combatexposed war veterans considered to be at risk of suicide with that in those not deemed to be at risk. Both groups had similar levels of depression and post-traumatic stress disorder. Members of the suicidal group showed stronger activation of the anterior cingulate and prefrontal cortex when they made mistakes while performing a concentration task. These brain areas are involved in cognitive control and monitoring of actions. The authors hypothesize that the extra effort used to process errors during self-monitoring may represent a vulnerability that could dent people's ability to cope with stress. This deserves investigation in larger samples, taking into account different emotional states.

Fund suicide research. Governments and funding agencies should invest more in the topic. The Horizon 2020 European Union Framework Programme for Research and Innovation should include a challenge devoted to suicide research - most urgently, the definition of valid criteria for suicidal behaviour as a mental disorder and the investigation of putative abnormalities in emotion-regulation circuits associated with such behaviour. The Societal Challenges presently defined in the framework do not mention suicide. The US National Institute of Mental Health in Bethesda, Maryland, has requested applications to develop ways to screen adolescents for suicide risk, but larger and more comprehensive programmes are necessary.

Promising in this regard is the institute's Research Domain Criteria project, which offers funding for the development of ways to classify psychopathology according to observable behaviour and neurobiological measures. For example, apathy is frequently observed in psychiatric and neurological disorders such as depression, schizophrenia, Parkinson's disease and Alzheimer's disease. Because the mechanisms leading to apathy may well be the same across these disorders, apathy is increasingly being studied as a distinct syndrome, irrespective of whether the patient has other symptoms of psychiatric or neurological disorders. Because suicide is a risk in various mental disorders, and encompasses neurobiological as well as social aspects, it is similarly suitable as topic within this project.

Promote prevention. Governments should invest as much in suicide prevention as they do in reducing fatal road accidents. In 2008-09, UK spending on road-safety awareness, including television advertisements, topped $£ 19$ million (US $\$ 32$ million); by contrast, $£ 1.5$ million was invested over 


\section{PREVENTION PAYS}

Economic modelling predicts that in one year, the costs of training UK general practitioners in suicide prevention, and the ensuing costs of psychological and pharmaceutical therapy they may prescribe to at-risk people, are outweighed by the savings to the public purse owing to the roughly 600 deaths averted.

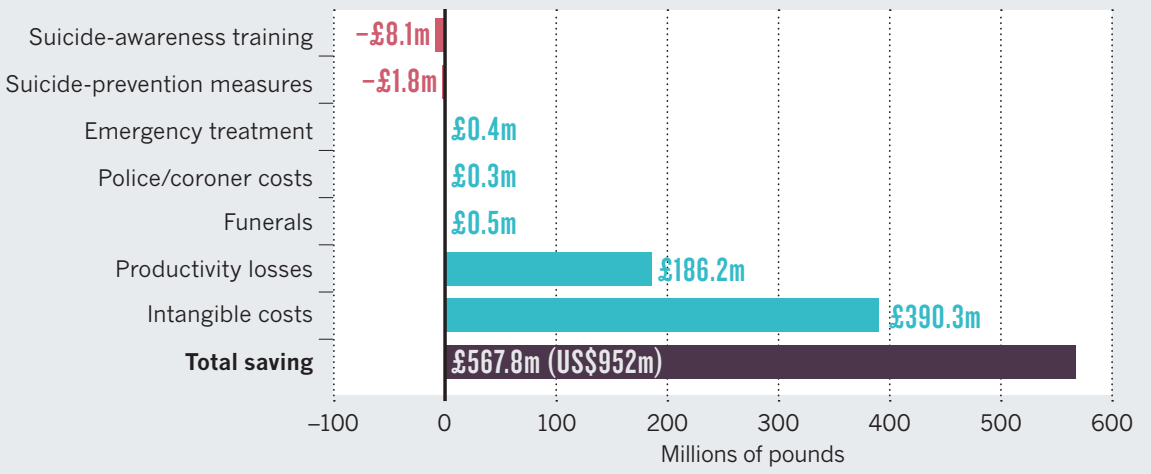

three years in suicide research. Fatal road accidents have declined steadily over the past decades, whereas suicide rates have levelled or even increased. Suicide awareness and prevention was highlighted in a review published earlier this year, which concluded that there is a return on investment for several mentalhealth promotion and illness-prevention interventions ${ }^{8}$ (see 'Prevention pays').

Risk factors are known from epidemiological studies, which should help to shape developing programmes aimed at preven-

\section{"Governments} should invest as much in suicide prevention as they do in reducing fatal road accidents." tion. Notable risk factors are mental disorder, previous suicide attempt, anxiety, impulsivity in combination with aggressive tendencies, family history of suicide and stressful life events such as job loss or divorce. Comprehensive prevention programmes should be developed that incorporate state-of-the-art knowledge ${ }^{3}$.

A good prevention programme would increase awareness and mental-health literacy in the general population to improve people's understanding of warning signs. Better education is also important for general practitioners (GPs), because many people with suicidal thoughts contact their GP in the weeks before attempting suicide. Prevention programmes would offer clear and easy access points for help, and a monitoring service for those at risk. Programmes must enlist governments and other stakeholders to tackle stigma, a major obstacle to suicide prevention.

Few have systematically implemented such programmes. Examples include Finland, Scotland and the US military. Their efforts should now be evaluated to pave the way for evidence-based improvements.

Coordinated efforts are clearly needed from public-health authorities, clinicians and scientists to understand and prevent suicide. Researchers should take advantage of progress in neurobiology and neuroimaging technology to uncover the brain mechanisms involved. Clinicians must focus on suicidal behaviour as a target of treatment in its own right. As Australia's National Mental Health Commission put it": "We can and must do better."

André Aleman is professor of cognitive neuropsychiatry at the University of Groningen, the Netherlands. Damiaan Denys is professor of psychiatry at the University of Amsterdam, and is at the Netherlands Institute for Neuroscience, Amsterdam, the Netherlands. e-mails:a.aleman@umcg.nl; d.denys@amc.uva.nl

1. Sadock, B. J., Sadock, V. A. \& Ruiz, P. (eds) Kaplan and Sadock's Comprehensive Textbook of Psychiatry 7th edn 2031-2040 (Lippincott Williams \& Wilkins, 2001).

2. Nock, M. K. et al. PLoS Med. 6, e1000123 (2009).

3. World Health Organization Public Health Action for the Prevention of Suicide: A Framework (WHO, 2012).

4. Pisani, A. R. et al. J. Youth Adolesc. 42, 807-820 (2013).

5. van Heeringen, C., Bijttebier, S. \& Godfrin, K. Neurosci. Biobehav. Rev. 35, 688-698 (2011).

6. Dour, H. J., Cha, C. B. \& Nock, M. K. Behav. Res Ther 49, 294-298 (2011)

7. Matthews, S., Spadoni, A., Knox, K., Strigo, I. \& Simmons, A. Psychosom. Med. 74, 471-475 (2012).

8. Research Prioritization Task Force A Prioritized Research Agenda for Suicide Prevention: An Action Plan to Save Lives (Action Alliance for Suicide Prevention, 2014).

9. Lourey, C., Plumb, J. \& Mills, A. A Contributing Life: The 2013 National Report Card on Mental Health and Suicide Prevention (National Mental Health Commission, 2013).

\section{CORRECTION}

The Comment 'Realizing China's urban dream' (Nature 509, 158-160; 2014) wrongly stated that more than 75.8 million hectares of arable land in China could be realized by optimizing rural residential land use. The correct figure is 7.58 million hectares. 